\title{
A further study of short-term retention and presentation rate'
}

\author{
M. S. MAYZNER AND K. M. SCHOENBERG
}

NEW YORK UNIVERSITY

\begin{abstract}
Abstraet
Six groups of $10 \mathrm{Ss}$ each were tested in a $3 \times 2 \times 4$ mixed analysis of variance design, in which the effects of 3 variables, namely, list length $(10,14$, and 18 digits), number of cycles (single and multi-cycle), and presentation rate $(4,2,1$, and .5 sec. per digit), on the short-term retention of a string of digits were examined. The results clearly showed that all 3 variables produced systematic effects on short-term retention and it was concluded that a trade off between number of cycles and presentation rate is possible, if total display time is held constant, thus supporting the previous work of Bugelski and Murdock in this area.

\section{Problem}

In a previous study (Mayzner \& Schoenberg, 1965) which examined the effects of presentation rate on short-term retention Ss were presented with lists of numbers 18 digits in length, one digit exposed at a time on a memory drum, at four different presentation rates, i.e., $4,2,1$, and $.5 \mathrm{sec}$. per digit. The $4 \mathrm{sec}$. rate was presented for 1 cycle, the 2 sec. rate for 2 cycles, the 1 sec. rate for 4 cycles, and the $.5 \mathrm{sec}$. rate for 8 cycles, thus total display time was held constant (72 sec.) for all four presentation rates. The results indicated that short-term retention decreased as presentation rate increased, even with total display time held constant.

The above results are somewhat at variance with results obtained by Bugelski (1962) and Murdock (1960) who, in general, found total display time to be more critical than presentation rate, however, neither of these studies were concerned with the short-term retention of a string of digits. Most recently Murdock (1965) suggests that "one should be able to trade off number of presentations and presentation rate if total presentation time is held constant," and he finds reasonable support for this hypothesis. The present study was designed therefore to examine this trade off function in more detail, particularly as it relates to the short-term retention of a string of digits.

\section{Method}

A Lafayette memory drum (Model 303B) was used to present the stimulus material. For the three different list lengths $(10,14$, and 18 digits), 16 separate lists were generated randomly. A $3 \times 2 \times 4$ mixed analysis of variance design (Edwards, 1960, p. 233) was employed with 10 different Ss assigned randomly to each of the six main conditions (i.e., 3 list lengths combined with 2 cycle conditions), with 16 trials or lists per S per condition. List length was examined at 3 levels $(10,14$, and 18 digits per list), number of cycles was examined at 2 levels (multi-cycle and single cycle), and presentation rate was examined at 4 levels $(4,2,1$, and .5 sec. per digit).

In the single cycle conditions each $\mathrm{S}$ received 16 separate lists as follows: List $1-4$ sec. per digit, List $2-2$ sec. per digit, List $3-1$ sec. per digit, List $4-.5$ sec. per digit, List $5-4$ sec. per digit, List $6-2$ sec. per digit... List $16-.5$ sec. per digit. Thus, each $S$ received 4 lists at each of the 4 presentation rates (a total of 16 lists) always in the same fixed order. At the completion of each of the 16 separate lists $\mathrm{S}$ was required to recall the list just presented to him, his scores on the 4 lists for each of the 4 different presentation rates were summed, converted to percent correct, and then employed as the dependent response measure for the analysis of variance and the functions shown in Fig. 1.

In the multi-cycle conditions the same procedure was followed as for the single cycle conditions, with one exception, namely, the number of cycles was varied (i.e., the number of times a given list was repeated to $S$ before recall of that list was requested) in order to keep total display time constant for all four presentation rates. This was accomplished, in the same manner as in a previous study (Mayzner \& Schoenberg, 1965), by presenting the 2,1 , and .5 sec. per digit rates for 2,4 , and 8 cycles, respectively. The $4 \mathrm{sec}$. per digit rate is, of course, presented for just 1 cycle in both the single and multi-cycle conditions. As in the previous study by Mayzner \& Schoenberg (1965) a red line was used to separate cycles, alerting $S$ that the sequence of digits that he had just observed one at a time in the window of the memory drum was about to repeat itself. Also, $S$ was informed of the number of cycles to be employed for his particular condition.

\section{Results and Diseussion}

Figure 1 presents the percent correct recall as a function of the various experimental conditions being studied, i.e., 3 different list lengths, single and multicycles, and 4 different presentation rates. Examination of Fig. 1 clearly shows the systematic effects of these variables on short-term retention. The results of the analysis of variance, in which the percent correct scores were transformed to inverse sine value as recommended by Edwards (1960, p 130), are as follows: (1) significant F-values of $14.10,16.56$, and $25.17(p<.001)$ were obtained for the three main effects, list length, number of cycles, and presentation rate, respectively, (2) one significant $F$-value of $8.52(p<.001)$ was obtained for the 


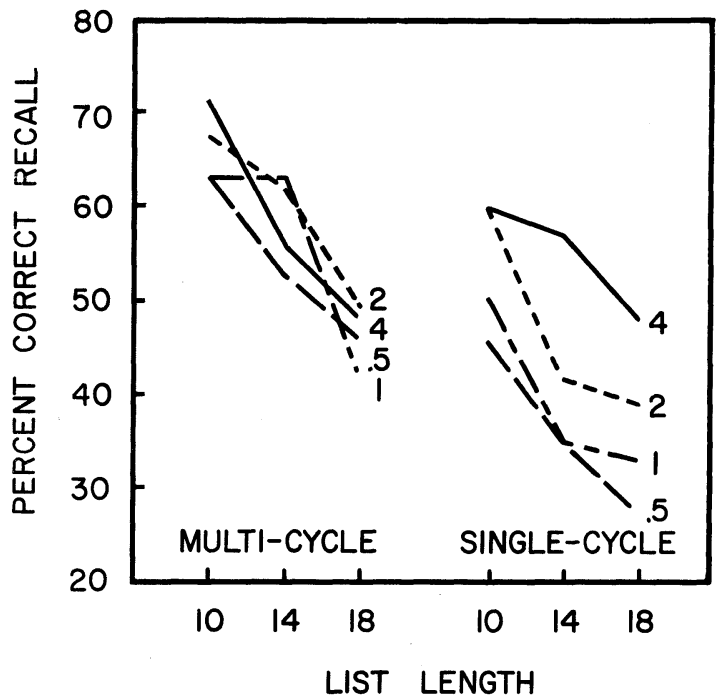

Fig. 1. Percent correct recall as a function of list length (10, 14 , and 18 digits), number of cycles (multi-cycle and single cycle), and presentation rate $(4,2,1$, and .5 sec. per digit).

cycle-presentation rate interaction, with the two remaining interaction terms yielding insignificant Fvalues, and (3) one significant $F-$ value of $4.30(p<.001)$ was obtained for the triple interaction term.

The functions shown in Fig. 1 combined with the results obtained from the analysis of variance reveal the following findings. (1) Short-term retention shows significant decrements for all four different presentation rates under both the single and multi-cycle conditions as list length increases from 10 to 18 digits. (2) The multi-cycle condition leads to superior short-term retention in contrast to the single cycle condition for the various list length and presentation rate combinations. (3) Under the single cycle condition increases in presentation rate from $4 \mathrm{sec}$. per digit to $.5 \mathrm{sec}$. per digit produce systematic decrements in short-term retention supporting previous findings by Macworth (1962). (4) Under the multi-cycle condition, where total display time is held constant for the four different presentation rates increases in presentation rate from 4 sec. per digit to .5 sec. per digit do not produce systematic decrements in short-term retention. This last finding is at variance with previous work by Mayzner \& Schoenberg (1965), but is in complete agreement with previous results obtained by Bugelski (1962) and Murdock $(1960 ; 1965)$.

Because of the discrepancy in results obtained for the multi-cycle condition, four additional groups of $10 \mathrm{Ss}$ each were examined under multi-cycle conditions as follows: Groups 1 and 2 received 8 trials of 10 digits at either the 4 or .5 sec. per digit rate and Groups 3 and 4 received 8 trials of 18 digits at either the 4 or .5 sec. per digit rate. The mean number of digits correctly recalled summed over the 8 trials, following the procedure used in the previous study by Mayzner \& Schoenberg (1965), for Groups 1 and 2 were 77.8 and 74.1 digits, respectively, and the mean number of digits correctly recalled summed over the 8 trials for Groups 3 and 4 were 78.1 and 78.8 digits, respectively. No significant differences were found between either Groups 1 and 2 or between Groups 3 and 4, again supporting the previous findings of the present study and the work of Bugelski and Murdock, but at variance with the earlier findings of Mayzner \& Schoenberg (1965), which found means of 76.7 and 62.9 for the number of digits correctly recalled for the 18 digit list at the 4 and $.5 \mathrm{sec}$. per digit rates, respectively.

In view of the evidence now available it seems reasonable to conclude that, when total display time is held constant, a trade off between the number of presentations or cycles and presentation rate is possible, not only for paired-associate material as shown by Bugelski and Murdock, but also for the short-term retention of a string of digits, as shown in the present study. However, the boundary conditions for which such trade offs are possible, with respect to number of cycles and presentation rate, are yet to be defined and work is presently in progress directed to this issue. In fact, the discrepancy in results between the present study and the previous study by Mayzner \& Schoenberg (1965) may be attributable in part, at least, to special effects which may occur as the boundary conditions of the phenomenon are approached. For example, if presentation rate is increased further from the $.5 \mathrm{sec} .-8$ cycle rate to the $.25 \mathrm{sec} .-16$ cycle rate to the $.125 \mathrm{sec} .-32$ cycle rate, a point should be reached where a trade off would no longer be possible, since presentation rate would begin to interfere with a "clear" perception of the stimulus series itself. If such effects begin to appear in the region of the $.5 \mathrm{sec} .-8$ cycle rate to the $.25 \mathrm{sec}$. -16 cycle rate then discrepancies between studies might possibly result, particularly if the boundary conditions are sensitive to individual difference factors in perceptual or reading speed and a particular boundary condition becomes disproportionately loaded with respect to this factor.

\section{References}

Bugelski, B. R. Presentation time, total time, and mediation in paired-associate learning. J. exp. Psychol., 1962, 63, 409-412.

Edwards, A. L. Experimental design in psychological research. (Red. ed.) New York: Rinehart, 1960.

Mackworth, J. F. Presentation rate and immediate memory. Canad. J. Psychol., 1962, 16, 42-47.

Mayzner, M. S., \& Schoenberg, K. M. Short-term retention and presentation rate. Psychon. Sci., 1965, 2, 111-112.

Murdock, B. B., Jr. The immediate retention of unrelated words. $J$. exp. Psychol., 1960, 60, 222-234.

Murdock, B. B., Jr. A test of the "limited capacity" hypothesis. J. exp. Psychol., 1965, 69, 237-240.

\section{Note}

1. This research was supported in part by Contract Nonr 285(56) between the Engineering Psychology Branch of the Office of Naval Research and New York University. 